\title{
Frontières
}

\section{La lumière des lilas}

\section{Marité Villeneuve}

Volume 20, numéro 1, automne 2007

La « bonne mort»

URI : https://id.erudit.org/iderudit/017955ar

DOI : https://doi.org/10.7202/017955ar

Aller au sommaire du numéro

Éditeur(s)

Université du Québec à Montréal

ISSN

1180-3479 (imprimé)

1916-0976 (numérique)

Découvrir la revue

Citer cet article

Villeneuve, M. (2007). La lumière des lilas. Frontières, 20(1), 93-96.

https://doi.org/10.7202/017955ar

Ce document est protégé par la loi sur le droit d'auteur. L'utilisation des services d'Érudit (y compris la reproduction) est assujettie à sa politique d'utilisation que vous pouvez consulter en ligne.

https://apropos.erudit.org/fr/usagers/politique-dutilisation/
Cet article est diffusé et préservé par Érudit.

Érudit est un consortium interuniversitaire sans but lucratif composé de l’Université de Montréal, l'Université Laval et l'Université du Québec à Montréal. Il a pour mission la promotion et la valorisation de la recherche. https://www.erudit.org/fr/ 


\section{LA LUMIÈRE DES LILAS}

Marité Villeneuve, M. Ps., écrivaine.

Un soleil mauve a saupoudré ta mémoire de cendres j'ai vu les dernières oies traverser ton regard (Villeneuve, 2000)

Maman a perdu la mémoire et moi j'ai perdu ma mère. Je l'ai perdue à petits feux, bien avant qu'elle ne parte à jamais. Je l'ai perdue peu à peu, à partir du jour où la maladie d'Alzheimer s'est installée dans sa vie.

Aussi écrirai-je ce témoignage comme une valse à deux temps : ce qu'a pu représenter, pour ma mère d'abord, le deuil de sa mémoire - ou du moins ce que j'ai pu en comprendre - et, dans un deuxième temps, ce qu'a signifié pour moi, en tant qu'aidante naturelle, accompagner la mémoire qui s'en va, avec les deuils qui en découlent.

\section{PERDRE LA MÉMOIRE}

Comment parler d'un deuil que l'on n'a pas vécu soi-même? Comme tout le monde, je connais la frustration de perdre mes clés de temps à autre pour les découvrir cinq minutes après dans la poche d'un manteau; l'impatience de ne pas retrouver le titre d'un film que j'ai vu récemment; le malaise de rencontrer une personne dont j'ai oublié le nom et le recours à un stratagème pour éviter que cela ne paraisse; la panique toute proche si, dans un endroit public, je crois pendant un instant mon sac à main disparu... Je connais ces expérien-

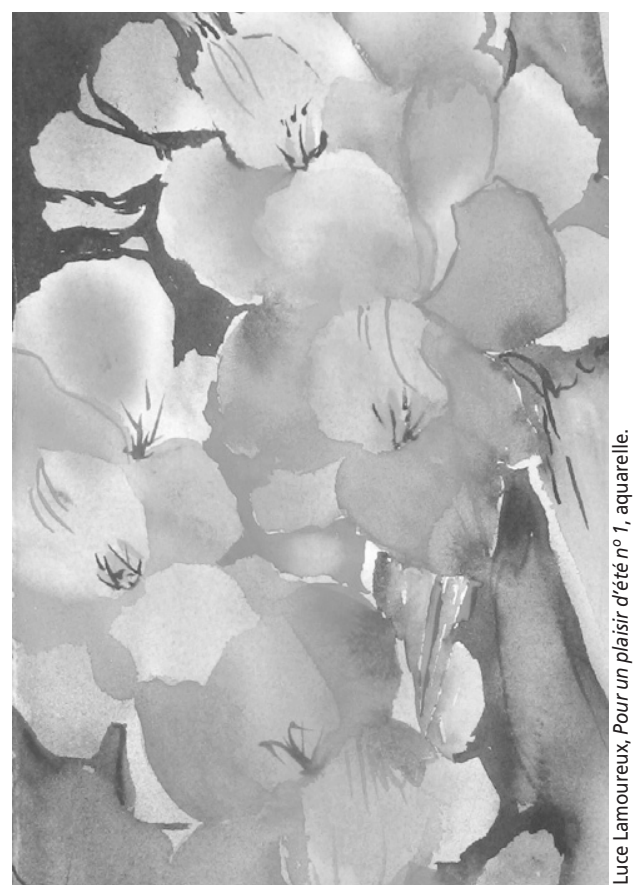

ces occasionnelles et le désagrément qui les accompagne. Mais quand cela devient un phénomène habituel, quand voir s'en aller sa mémoire, et ce, de façon irréversible, devient une épreuve quotidienne, je ne peux qu'imaginer l'angoisse et le sentiment de catastrophe générés par cet état.

\section{LA CONSCIENCE DOULOUREUSE}

J'ai accompagné ma mère sur le chemin de cette perte pendant plusieurs années. J'ai eu la «chance» d'avoir une mère qui a conservé longtemps sa lucidité ainsi que la conscience de ses déficits et qui s'est exprimée verbalement pendant presque toute la durée de la maladie. Je prends soin d'écrire le mot «chance» entre guillemets, car si cela m'a permis, à moi, de recueillir cette parole précieuse pour en faire aujourd'hui un témoignage, je crois que cette conscience aiguë et persistante de l'effritement de sa mémoire fut pour ma mère l'un des aspects les plus douloureux de son état.

Je revois tous les petits bouts de gommette bleue qui tapissaient les murs du dernier appartement où elle a vécu seule, avant que la maladie ne soit diagnostiquée. Elle collait tout sur les murs pour ne pas oublier: le numéro de téléphone d'une voisine, son prochain rendez-vous avec le médecin, une course à faire, la date d'une transaction financière à effectuer, etc. Et même quand les rendez-vous étaient périmés, elle n'enlevait pas les mémos, si bien que les murs en étaient couverts. Peut-on réaliser tout l'effort, toute l'énergie que cela représente: se dire constamment «il ne faut pas que j'oublie»?

C'était avant le diagnostic. J'attribuais alors ce comportement à un trait de caractère de ma mère. Efficace et organisée, elle avait l'habitude d'afficher son horaire et d'autres mémos importants, au temps où elle était enseignante. J'ai accusé aussi la solitude, l'anxiété, le sentiment d'abandon que lui faisait vivre la mort récente de mon père (les troubles s'étant manifestés tout de suite après). J'ai soupçonné la dépression. Ou une manie qui peut-être s'amplifiait avec le vieillissement... Au départ, on ne comprend pas très bien ce qui se passe. Mais le jour où je l'ai vue se perdre dans le centre commercial qu'elle fréquentait depuis toujours, j'ai été très inquiète.

Qui s'est déjà perdu dans une ville étrangère, dans une langue étrangère, sait le désarroi et le sentiment de vulnérabilité que l'on peut éprouver. Un jour que je suggérais à maman de se faire raccompagner, alors qu'elle venait de s'égarer dans la vaste résidence pour personnes âgées où elle a vécu quelque temps, elle m'a répondu: «Si je demande le chemin, on va voir que la vieille, elle n'a plus toute sa tête...» J'ai compris à quel point la conscience de ses déficits 
la faisait se sentir vulnérable et en danger d'abus. Je la revois trottinant dans le corridor, le sac à main collé contre son corps.

\section{VIVRE EN EXIL}

Avec la progression de la maladie, les lacunes de la mémoire envahissent le domaine des mots. Et perdre le langage, c'est comme être en exil. C'est par le langage que nous découvrons notre monde intérieur, que nous construisons notre identité. C'est par le langage que nous nous relions aux autres. Ne pas trouver les mots pour exprimer ses besoins ou pour répondre à une personne qui nous adresse la parole, c'est un peu comme se retrouver dans un pays dont on ne maîtrise pas la langue. L'écrivain d'origine chinoise François Cheng, qui a dû apprendre une langue seconde lorsqu'il est arrivé dans son pays d'adoption, la France, décrit bien cette souffrance. En plus du sentiment d'abandon et de dénuement que connaît tout exilé, écrit-il, ce dernier éprouve une souffrance plus «humiliante» encore, celle de se voir rabaissé aux yeux des autres. «Baragouinant des mots ou des phrases parfois approximatifs, incapable d'un récit clair et cohérent, il donne l'impression d'être dépourvu de pensées, voire de sentiments»(Cheng, 2002, p. 28-29).

Ma mère éprouvait cette sensibilité au regard d'autrui; elle craignait le ridicule et les moqueries. L'effort pour trouver ses mots, les difficultés devant une conversation la portaient à se couper des autres et à se replier sur elle-même. Elle se sentait humiliée de ne plus savoir lire, alors qu'elle avait été professeur: "Surtout ne le dis à personne!» Je la surprenais parfois à essayer de recopier des mots, des bouts de phrase dans un cahier. Je l'ai vue se promener avec son nom dans sa poche et, pendant longtemps, elle a trimballé sa mallette d'enseignante contenant des trésors personnels, comme d'inséparables morceaux de mémoire.

\section{LA NOSTALGIE DU RETOUR}

Est-ce en réponse à ce sentiment d'exil intérieur que tant de personnes atteintes d'Alzheimer veulent rentrer à la maison, demandant parfois à retourner sur les lieux de leur enfance? Je ne saurais le dire. Si ma mère n'était pas de celles qui «fuguaient» (un mot qui me rebute en raison de sa charge négative et de la généralisation qu'il comporte), elle n'a jamais cessé par ailleurs, tout au long de la maladie, de vouloir rentrer à la maison, vivre avec ses enfants et sa famille. Le jour où je l'ai emmenée dans notre région d'origine pour visiter une de ses sœurs et qu'elle ne l'a pas reconnue, j'ai compris que ce chez-soi perdu et recherché était désormais un lieu intérieur, une mémoire qui subsiste bien
EN LA RACCOMPAGNANT

EN VOITURE, ELLE M'A DIT:

«JE VOULAIS TE DIRE

QUELQUE CHOSE... C'EST ÇA

C'EST LA DÉTRESSE

DE MON IMPUISSANCE... ॥

après que tout le reste a été oublié : la chaleur des premiers liens et de l'intimité.

Je l'ai vue marcher pendant des jours, encore et encore, marcher du matin au soir, arpenter le long corridor entre la cuisine de la résidence et sa chambre, sa petite valise à la main. Marcher pour conserver son autonomie; marcher pour contrer l'anxiété, mais aussi parce qu'on va peutêtre bientôt rentrer chez soi. Cette volonté de rester debout, de conserver sa dignité, de conserver ce qui la reliait au reste du monde et surtout aux siens, aux êtres les plus importants de sa vie, cette volonté-là ne l'a jamais quittée.

Un jour que nous étions attablées dans un restaurant et qu'elle venait de me raconter ses peines reliées à la maladie (c'était dans les débuts), touchée par ses propos, je lui ai dit: "Ce que tu m'apprends est précieux; je veux écrire un livre pour aider d'autres personnes. » Dans un élan spontané, maman m'a répondu: "Oh oui! Alors, pose-moi des questions, je vais te répondre!» Elle était de celles qui avait toujours soutenu les autres dans l'épreuve. Elle était fière de pouvoir partager ce qu'elle vivait. Se sentir encore utile et reliée à d'autres humains l'a peut-être aidée à passer au travers de son propre désastre. Je crois que cela peut soutenir la vie, même dans des conditions de profonde détresse.

\section{ÊTRE UNE PERSONNE}

Il est bon de rappeler que la maladie d'Alzheimer est une maladie physique au départ. Il s'agit d'atteintes neurologiques qui affectent progressivement la mémoire, l'orientation, le jugement et le langage et qui ont des répercussions sur la vie affective et sociale. Ce lent processus de détérioration au cours duquel la personne va de perte en perte, de détachement en détachement, s'accompagne généralement de détresse morale ou l'amplifie, quand la personne était déjà fragile. Perdre du pouvoir sur sa vie, son sentiment de sécurité, son indépendance (souvent symbolisée par l'auto), dans bien des cas devoir quitter sa maison, se séparer des siens, soulèvent des réactions psychologiques normales. J'ai pu constater qu'on associe trop facilement maladie d'Alzheimer et troubles du comportement. Ces derniers, quand ils se manifestent (ce qui n'est pas toujours le cas), sont bien souvent une forme d'expression de l'angoisse, une façon de réagir au sentiment d'être diminué, rabaissé, ou au contrôle (parfois excessif) exercé sur les personnes, même s'il s'agit par là de les protéger.

Au cours d'un examen de routine visant à mesurer le degré d'atteintes cognitives (ce type d'évaluation où l'on vous pose des questions du genre " où sommes-nous, dans quelle ville, en quelle saison, etc.», maman a répondu à l'infirmière qui l'interrogeait: «T'es qui, toi, une petite jeune comme ça, pour me poser des questions aussi insignifiantes?»

À partir du moment où un diagnostic est posé, on ne voit plus la personne dans son ensemble, avec ses qualités, ses compétences, son histoire. On ne voit que la maladie et des symptômes. Quant à la personne, elle devient une «malade». Maman cherchait désespérément à sortir de ce rôle de «malade» dans lequel elle se sentait enfermée et à conserver sa dignité, ce qui, parfois, pouvait prendre des allures de «troubles du comportement». Elle réagissait mal quand on lui imposait des activités de manière trop rigide; devant l'obligation, par exemple, de se soumettre à un bain, d'avaler des médicaments amers, etc. Elle disait: «Mon corps m'appartient... Ils me prennent pour un enfant...»

Je voyais son regard triste quand, assise sur la galerie de la résidence, elle regardait les autres monter et descendre des voitures, aller et venir librement dans leur vie. Un jour que je l'avais emmenée chez moi et qu'elle me regardait travailler, elle me dit: "Je te regarde toi, tu es jeune et efficace, moi, je ne sais plus rien faire...» En la raccompagnant en voiture, elle m'a dit: "Je voulais te dire quelque chose... c'est ça... c'est la détresse de mon impuissance...»

Elle exprimait par moments une envie de mourir: " Est-ce que tout cela va bientôt finir?» Et d'autres fois: "C'est pas pour tout de suite... J'aime ça, moi, voir mes enfants... Ça me fait chaud au cœur... Au moins, j'ai encore mes jambes...»

Car, à travers tout ce long processus, la vie continue. Cela aussi, il faut le préciser. La vie ne finit pas avec l'annonce du diagnostic. Plusieurs années peuvent encore s'écouler, parsemées de moments heureux. J'ai vu maman rire, danser, faire des steppettes pour amuser les préposées, déguster une glace, regarder un écureuil autour d'une table à pique-nique, ici et maintenant trouver le jour beau, le soleil bon, sentir dans son cœur de la joie... Des «petits bonheurs» comme ceux-là, nous en avons vécu ensemble, et beaucoup. 


\section{ACCOMPAGNER LA MÉMOIRE QUI S'EN VA}

\author{
Pour égayer tes jours je recouds \\ des lambeaux de lumière j'arrache \\ au ciel ses colliers d'outardes
}

(Villeneuve, 2000)

Quand j'ai appris que ma mère souffrait de déficits cognitifs de type Alzheimer liés, dans son cas, à des atteintes cardiovasculaires, ce fut pour moi un choc: tout, mais pas cela! Pas cette maladie dégénérative avec la longue descente qui s'ensuit! «Ma mère ne peut pas avoir ça!» Sa mère à soi... ce n'est jamais pareil!

\section{AIDANT «NATUREL »?}

Voir se détériorer jour après jour un être cher est une expérience bouleversante, par moments, insoutenable. Certains choisissent, consciemment ou inconsciemment, de s'éloigner: parce qu'incapables de supporter cette réalité, parce qu'ils croient que ça ne vaut pas la peine de continuer puisque la personne a perdu la mémoire... Pour ma part, j'ai choisi de m'engager auprès de ma mère, d'assumer cet accompagnement, sans savoir où cela me conduirait.

Quel paradoxe que ce terme d'aidant «naturel», comme si cela était naturel de sacrifier une partie de sa vie pour accompagner un proche dans une longue maladie! D'autant plus paradoxal qu'il arrive même que l'on soit plus ou moins "désigné» comme aidant, parce qu'on est la personne la plus disponible de la famille, ce qui était un peu le cas pour moi. Et même si j'ai choisi d'assumer cet accompagnement, même si une part de ce choix vient d'un élan «naturel», au sens d'un mouvement émanant du cœur, poussé par un lien d'attachement avec l'autre, il reste qu'une certaine perte de liberté découle de cet engagement et cette perte n'a rien de «naturelle». La nature humaine désire le plaisir et cherche à éviter la souffrance: voilà le mouvement «naturel»! Côtoyer quotidiennement la souffrance, sentir la dépendance d'une autre personne envers soi, l'assister et la soutenir, devoir prendre des décisions à sa place entraînent nécessairement des responsabilités très lourdes à porter et des contraintes à la liberté. Cela prend aussi du temps, de l'écoute, de la patience... Et la vie, elle, continue de tourner avec son quotidien exigeant.

L'accompagnement m'a peu à peu isolée socialement, sans que je m'en aperçoive au départ. Parce que j'étais trop fatiguée pour sortir et rencontrer des amis; parce que la problématique m'envahissait parfois à un point tel que je ne me sentais plus sur la même longueur d'ondes que les autres: le cinéma, les restaurants, la vie extérieure, c'était bien loin tout ça! Il m'a semblé pendant un certain temps n'avoir plus qu'un seul sujet de conversation: l'Alzheimer!

\section{UNE SUCCESSION DE PETITS DEUILS}

Accompagner une personne souffrant d'une maladie dégénérative, cela signifie au quotidien vivre une succession de petits deuils. J'ai pleuré ce jour où il m’a fallu enlever nos portraits de famille sur les murs parce que maman avait peur de ces «étrangers» qui la regardaient... J'ai été remuée ce jour où elle m'a demandé si j'avais un père et une mère, moi... Et ce jour où elle a trouvé mon prénom bien joli, sa main évoquant quelque chose dans le vague: "Il y a quelqu'un par chez nous qui s'appelait comme ça... » Et cette première fois où j'ai dû la déplacer en fauteuil roulant... Cette première fois où j'ai dû l'aider à manger...

J'ai vécu jour après jour l'épreuve du «renoncement». Renoncer à un irrationnel désir de toute-puissance (je ne peux rien faire pour guérir maman), au sentiment illusoire d'être unique (le jour où je suis devenue un visage parmi les autres). Renoncer aux certitudes rassurantes (comment savoir si une décision que l'on prend est la bonne), à la volonté de changer le système quand on se retrouve devant la grosse machine institutionnelle...

Je me suis cognée le nez aux paradoxes de «l'acceptation». "Accepter» (mais est-ce bien le mot?) un «placement» pour sa mère, une médication qui soulage la douleur mais en même temps accentue les déficits. "Accepter» un événement que l'on n'a pas voulu, pas choisi, et qui s'impose comme un intrus dans notre vie. «Accepter» l'injustice d'un destin et de l'inégale répartition des épreuves (ma mère n'avait-elle pas déjà eu son lot?), etc.

Bref, si maman s'est battue pendant des années pour conserver son autonomie et sa dignité, je ne fus pas non plus à l'abri des combats, des tumultes et des contradictions. Accompagner, c'est faire le deuil d'une relative et bien fragile paix.

Mais n'est-ce que cela? Cette somme de difficultés et d'épreuves? Alors pourquoi le ferait-on? Pourquoi s'engagerait-on sur un chemin qui durera peut-être des années? $\mathrm{Y}$ a-t-il des gains sur cette route?

\section{DES GAINS}

J'étais de celles qui recherchaient les grandes choses. À quarante ans, je suis partie voyager autour du monde pendant un an. Dans les années d'accompagnement, j'ai voyagé entre deux maisons: la résidence où vivait ma mère et ma propre demeure... Quelques kilomètres suffisent parfois pour apprendre qu'il y a aussi du bonheur dans les petites choses et dans la continuité des liens. «Oui, maman, je suis là, je vais venir demain. » Aller marcher au bord du fleuve (quand cela lui était encore possible), s'asseoir sur un banc et regarder ensemble un bateau qui passe, un nuage, un oiseau... Ces moments resteront gravés dans ma mémoire tout au long de ma vie. Il m'a fallu ralentir mon rythme, apprendre les petits pas de la vieillesse, quand la vie vous dépasse à grandes enjambées; côtoyer la déchéance et la fragilité dans un monde où la force et la beauté sont reines.

J'ai vécu cet accompagnement comme une expérience réparatrice. Au cours de ces années, j'ai mieux connu ma mère. Jamais je n'avais été aussi proche d'elle depuis les lointains jours de l'enfance. Soigner sa mère, l'aider à se laver, à se coiffer, choisir avec elle ses vêtements, s'asseoir genoux contre genoux dans l'intimité de sa chambre, écouter ses chagrins, essayer de comprendre ses colères, d'apaiser ses craintes, de déchiffrer les mots qui s'effritent comme le biscuit que l'on partage; puis s'étendre auprès d'elle, vers la fin de sa vie, quand il n'y a plus de mots et qu'on est fatiguée, si fatiguée de cette longue route, qu'il n'y a plus rien d'autre à faire que de s'étendre auprès de maman et de s'endormir comme lorsqu'on était enfant... Ce sont des gestes qui rapprochent et qui ont contribué à réparer en moi des aspects d'une intimité perdue. J'ai été amenée à materner ma propre mère, moi qui n'ai jamais eu d'enfants!

Une amie très sensible au langage m'a fait remarquer que dans le mot «mémoire», il y a le mot «mère» et le mot «moi». En ramassant les cailloux perdus de sa mémoire, je me suis sentie ramenée chez moi.

\section{UNE CHAÎNE HUMAINE}

Nous formons une grande chaîne humaine. La main que je donne ici, quelqu'un d'autre me la tend ailleurs. La Société Alzheimer de Québec a constitué pour moi l'un des maillons de cette chaîne.

Quand un diagnostic aussi grave est posé, on se retrouve devant l'inacceptable, devant l'inconnu, devant un langage qui effraie et rebute (déficits, stades, chaise gériatrique, incontinence) et aussi devant une grosse machine administrative: CLSC, CHSLD, système de santé, évaluations et suivi, demandes d'hébergement, centres privés, centres publics, etc. On se sent bien démuni devant tout cela. Par où commencer? Les rencontres offertes par la Société Alzheimer m'ont aidée à mieux comprendre la maladie, à apprendre comment réagir devant certaines situations difficiles, à réaliser les enjeux sociaux reliés à la maladie, à défaire certains préjugés 
DANS LE SILENCE QUI ALLAIT PEU À PEU ENVELOPPER MA MÈRE,

DANS SON VOYAGE VERS LA NUIT PROFONDE,

JE ME SUIS SENTIE RELIÉE À QUELQUE CHOSE DE PLUS GRAND

QUE MOI. J'AI ÉTÉ PLACÉE DEVANT L'INCONNU,

LE MYSTÈRE, L'INSONDABLE.

qui l'accompagnent. L'information et le soutien reçus, notamment à travers les groupes d'entraide, me furent d'un grand secours, dans les premiers temps du moins. Pouvoir échanger avec d'autres personnes qui vivent des situations semblables m'a permis de me sentir moins seule, m'a parfois apporté des réponses, fait entrevoir des solutions.

J'ai eu la chance aussi de dénicher, dans le réseau privé, une petite ressource de type familial où ma mère a pu vivre pendant presque toute la durée de sa maladie, et qui fut pour moi comme une deuxième famille. Ce n'est jamais facile de "placer» sa mère (le mot même a une connotation négative), mais quand cela s'avère être la meilleure solution, la confiance dans le milieu choisi, la chaleur humaine qu'on peut y trouver et la possibilité de collaborer tous ensemble sont d'indiscutables soutiens.

L'écriture aussi m'a aidée, surtout en périodes de doute et de crise, quand je me sentais perdue, envahie et que j'avais besoin d'une bulle. Écrire m'a permis de restaurer ma bulle, de recréer mon espace intime. Ce fut ma façon personnelle de «rentrer chez moi». Pendant cinq ans, j'ai ainsi tenu un journal d'accompagnement. J'avais besoin de déposer sur la page les émotions que la maladie nous faisait vivre, ma mère et moi, mes questions, mes angoisses, mes révoltes, autant que mes découvertes et nos moments heureux.

Déposer tout cela pour prendre une distance, pour voir plus clair, mais aussi pour conserver la mémoire qui se délie, se détricote, comme si tricoter les mots était une façon de ramasser les mailles en train de se perdre. J'y ai réchappé des souvenirs d'enfance, j'y ai retricoté ma propre histoire et celle, en partie, de ma mère.

Et puis, l'écriture me fait aimer ma vie, m'aura fait aimer cette période quasiment hors du temps. Quand on écrit, on saisit des instants qui autrement seraient déjà oubliés, noyés dans la flux de la vie. Des moments difficiles, soit, mais aussi des instants de grâce et de beauté. Écrire me permet de mettre de l'ordre dans le chaos des événements et des pensées, parfois même de trouver du sens à ce qui, apparemment, n'en a pas. Si j'ai accompagné ma mère pendant toutes ces années, je peux dire que mes carnets d'écriture m'ont servi, à moi, d'accompagnants; ils ont été mes aidants naturels. La poésie m'a aussi montré la beauté du chemin (Villeneuve, 2000, 2005, 2007).

Des livres également m'ont soutenue et ressourcée quand je me sentais vidée. De ces livres qui nourrissent le cœur et l'esprit. De ceux qui sont porteurs de sens et d'espoir. De ceux dont on souligne des phrases et dont on saisit parfois un extrait pour le recopier dans son cahier ou l'afficher près de son lit. Ces livres-là font partie de la chaîne humaine dont je parlais plus haut. Ils sont notre héritage collectif. Des livres, mais plus que cela, des voix, m'ont accompagnée tout au long de ma vie et continuent de le faire.

\section{DE L'AUTRE CÔTÉ DE LA NUIT}

La maladie d'Alzheimer ressemble pour moi à la traversée d'un désert, avec son dépouillement progressif, son avancée vers le vide, vers la nuit.

Dans le silence qui allait peu à peu envelopper ma mère, dans son voyage vers la nuit profonde, je me suis sentie reliée à quelque chose de plus grand que moi. J'ai été placée devant l'inconnu, le mystère, l'insondable. J'ai vu le visage de ma mère s'illuminer par moments, j'ai senti son âme et la mienne s'agrandir. Mon cœur s'est ouvert. Suis-je plus sage que je ne l'étais auparavant? Saurai-je, le temps venu, me dépouiller à mon tour de mon être et de mes biens? Saurai-je me détacher plus facilement de ceux que j'aime? Je le voudrais bien, mais j'en doute. Je pense que partir, s'arracher au monde, se délester de soi-même est un processus permanent, commencé depuis notre naissance, un deuil toujours en cours, jamais achevé. Au mieux, je me sens peut-être un peu plus patiente, un peu plus aimante.

Cet accompagnement a soulevé chez moi des questions éthiques (fallait-il, au début de la maladie, lui poser ce stimulateur cardiaque qui allait prolonger ses jours?) ainsi que des angoisses métaphysiques... Et s'il existait "une nuit de l'âme», comme l'ont décrite certains mystiques... Si l'âme avait besoin de se désencombrer $\mathrm{du}$ fatras d'images qui l'envahit chaque jour, des informations qui la bombardent, de l'amoncellement de souvenirs entassés au cours d'une vie comme en un grenier trop encombré... Si l'âme avait besoin de ce dépouillement, de ce temps de repos, de cette avancée à petits pas... Et s'il y avait quelque chose de l'autre côté du désert, au-delà de la mémoire... Et si... Et si..

Je ne sais pas. Je ne sais rien de ces choses. Mais tant que l'on n'arrivera pas à "guérir » cette maladie, tant qu'il y aura des orphelins de la mémoire, alors qu'on adoucisse leur traversée! Que l'on fasse de cette nuit un lieu et un lit de repos. Qu'on l'entoure de bienveillance et d'amour. Qu'on se laisse toucher par le mystère qu'elle transporte. Qu'on lui laisse le temps!

J'emploie le mot "guérir» comme si l'on ne pouvait parler de maladie sans accoler à ce mot celui de guérison. Notre époque actuelle qui voue un culte à la beauté, à la performance et à l'éternelle jeunesse, qui ne tolère ni échec ni faiblesse ni déchéance, se rue vers toute promesse de guérison du moindre de ses maux. Or, «il n'est pas de blessure qui ne renvoie à la blessure d'Amour», pas de déchirure, pas de faille dans l'armure qui ne soit un éveil, un appel, et qui n'indique un chemin vers son humanité. "Cette fragilité est la musique de l'être, sa nudité essentielle et indestructible»(Kelen, 2005).

\section{LA LUMIÈRE DES LILAS}

Tes paysages émigrent en mes saisons j'entends ton rire dans la mémoire des fleurs (Villeneuve, 2000)

Ma mère aimait les lilas. Elle est décédée en juin 2003, en ce temps de l'année où ces arbres déploient leur plus belle floraison, leurs effluves et leurs couleurs veloutées. C'était aux premières lueurs de l'aube, de l'autre côté de la nuit.

Dans ma mémoire, les lilas sont toujours en fleurs.

\section{Bibliographie}

CHENG, F. (2002). Le Dialogue: Une passion pour la langue française, Paris, Desclée de Brouwer.

KELEN, J. (2005). Divine blessure, Paris, Albin Michel.

VILLENEUVE, M. (2007). Des pas sur la page. L'écriture comme chemin, Montréal, Fides.

VILLENEUVE, M. (2005). Je veux rentrer chez moi. Récit d'un accompagnement, Montréal, Fides (prix du «récit» AbitibiConsolidated 2005).

VILLENEUVE, M., (2000). Pays d'épaule et de mousse, poésie, Ripon, Écrits des HautesTerres. 